Please cite this paper as follows:

Chein-Shan Liu and Hong-Ki Hong, The Contraction Ratios of Perfect Elastoplasticity under Biaxial Controls, European Journal of Mechanics A/Solids, Vol.19, pp.827-848, 2000. 


\title{
The contraction ratios of perfect elastoplasticity under biaxial controls
}

\author{
Chein-Shan Liu ${ }^{\text {a }}$, Hong-Ki Hong ${ }^{b}$ \\ a Department of Mechanical and Marine Engineering, National Taiwan Ocean University, Keelung, 202-24, Taiwan \\ b Department of Civil Engineering, National Taiwan University, Taipei 106-17, Taiwan
}

(Received 16 July 1999; revised and accepted 22 February 2000)

\begin{abstract}
We studied contraction ratios, one rate form and one total form, of the Prandtl-Reuss model under combined axial and torsional controls. In the transition point of elasticity and plasticity, the rate form contraction ratio may undergo a discontinuous jump, which, depending on the control paths and initial stresses, may be positive, zero, or negative. For the total form contraction ratio, no similar jump phenomenon is observed in the elasticityplasticity transition point. Depending on initial stresses both ratios may be greater than $1 / 2$. In the simulations of the axial-torsional strain control tests, the hoop and radial strains are not known a priori and hence can not be viewed as inputs. This greatly complicates the constitutive model analyses because the resulting differential equations become highly non-linear. To tackle this problem, we devise a new parametrization of the axial and shear stresses, deriving a first order differential equation to solve for the parameter variable, with which the consistency condition and initial conditions are fulfilled automatically. For mixed controls, the responses can be expressed directly in terms of the parameter without solving the first order differential equation. In particular, when control paths are rectilinear exact solutions can be obtained. (C) 2000 Éditions scientifiques et médicales Elsevier SAS
\end{abstract}

perfect elastoplasticity / contraction ratio / plasticity / compressibility / biaxial control

\section{Introduction}

The combined axial and torsional testing of thin-walled tubes is ideal for the study of constitutive equations of metals; see, for example, Nadai (1950) and Hill (1950). The thin-walled tubular specimen is usually subjected to a combination of axial load $P(t)$ and torque $T(t)$, and with an appropriate feedback arrangement the length $Z(t)$ and the relative twist angle $\Theta(t)$ as well as $P(t)$ and $T(t)$ can serve as control variables. Thus the axialtorsional testing may have $(P, T),(Z, \Theta),(P, \Theta)$, and $(Z, T)$ as control input pairs, as illustrated in figure 1 . Under the assumption of uniform deformation and stress distribution in the main parallel segment of the thin wall of the specimen, the four control pairs can be correspondingly related to $\left(\sigma_{z z}, \sigma_{z \theta}\right),\left(\varepsilon_{z z}, \varepsilon_{z \theta}\right),\left(\sigma_{z z}, \varepsilon_{z \theta}\right)$, and $\left(\varepsilon_{z z}, \sigma_{z \theta}\right)$. They are, respectively, pure stress control, strain control, and mixed controls; see, for example, Klisinski et al. (1992). The stress and strain paths for the considered tests are such that their rates, or the tangents to the paths, are of the forms:

$$
\dot{\boldsymbol{\sigma}}=\left[\begin{array}{ccc}
\dot{\sigma}_{11} & \dot{\sigma}_{12} & 0 \\
\dot{\sigma}_{12} & 0 & 0 \\
0 & 0 & 0
\end{array}\right], \quad \dot{\boldsymbol{\varepsilon}}=\left[\begin{array}{ccc}
\dot{\varepsilon}_{11} & \dot{\varepsilon}_{12} & 0 \\
\dot{\varepsilon}_{12} & \dot{\varepsilon}_{22} & 0 \\
0 & 0 & \dot{\varepsilon}_{22}
\end{array}\right]
$$

where the superimposed dot denotes differentiation with respect to time $t, \mathrm{~d} / \mathrm{d} t$. Notice that $\dot{\sigma}_{21}=\dot{\sigma}_{12}$, $\dot{\varepsilon}_{21}=\dot{\varepsilon}_{12}$, and $\dot{\varepsilon}_{33}=\dot{\varepsilon}_{22}$. In cylindrical coordinates $(z, \theta, r)$ as shown in figure $1, \varepsilon_{11}=\varepsilon_{z z}$ is the axial strain, $\varepsilon_{22}=\varepsilon_{\theta \theta}$ is the hoop (or circumferential) strain, $\varepsilon_{33}=\varepsilon_{r r}$ is the radial strain, and $\varepsilon_{12}=\varepsilon_{z \theta}$ is the shear strain in the thin wall of the tube, whereas $\sigma_{11}=\sigma_{z z}$ is the axial stress and $\sigma_{12}=\sigma_{z \theta}$ is the shear stress in the thin wall of the tube. 


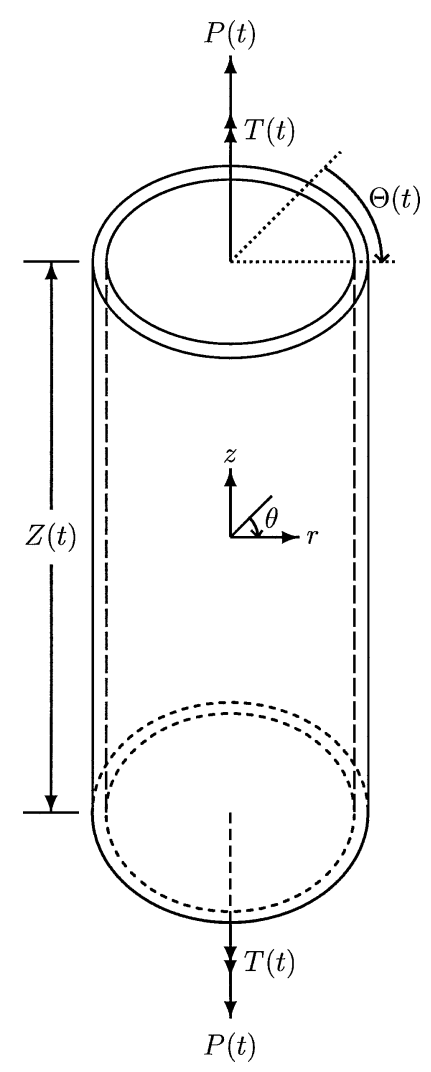

Figure 1. The main parallel segment of a thin-walled tubular specimen under the combined axial and torsional controls $(P, T),(Z, \Theta),(P, \Theta)$, or $(Z, T)$.

In any control case, $\dot{\varepsilon}_{33}=\dot{\varepsilon}_{22}$ is the quantity induced by the axial control on a compressible material and is not necessarily equal to zero ${ }^{1}$. We usually use the contraction ratios

$$
v_{r}:=\frac{-\dot{\varepsilon}_{22}}{\dot{\varepsilon}_{11}}, \quad v_{t}:=\frac{-\varepsilon_{22}}{\varepsilon_{11}}
$$

to measure the compressibility of the material, because

$$
\operatorname{tr} \dot{\boldsymbol{\varepsilon}}=\left(1-2 v_{r}\right) \dot{\varepsilon}_{11}, \quad \operatorname{tr} \boldsymbol{\varepsilon}=\left(1-2 v_{t}\right) \varepsilon_{11}+\varepsilon_{33}\left(t_{i}\right)-\varepsilon_{22}\left(t_{i}\right),
$$

where $t_{i}$ is an initial time. In definitions (2) the first is a rate form and the second is a total form. These two forms are different in the plastic phase but have the following relation:

$$
v_{r}=v_{t}+\varepsilon_{11} \frac{\mathrm{d} v_{t}}{\mathrm{~d} \varepsilon_{11}}
$$

\footnotetext{
${ }^{1}$ See the discussions following equations (16) or (56).
} 
Note that all the three quantities $v_{r}, v_{t}$ and $\varepsilon_{22}$ are functions of time $t$ and depend upon $\varepsilon_{11}$. There are two ways to derive the above formula: taking the time derivative of equation $(3)_{2}$, one obtains

$$
\operatorname{tr} \dot{\boldsymbol{\varepsilon}}=\left(1-2 v_{t}\right) \dot{\varepsilon}_{11}-2 \varepsilon_{11} \frac{\mathrm{d} v_{t}}{\mathrm{~d} \varepsilon_{11}} \dot{\varepsilon}_{11}
$$

where the chain rule was used in the derivative $\dot{v}_{t}$. Equating the above equation with equation (3) $)_{1}$, one obtains

$$
\left(1-2 v_{r}\right) \dot{\varepsilon}_{11}=\left(1-2 v_{t}\right) \dot{\varepsilon}_{11}-2 \varepsilon_{11} \frac{\mathrm{d} v_{t}}{\mathrm{~d} \varepsilon_{11}} \dot{\varepsilon}_{11},
$$

which, through some arrangement, yields equation (4) if $\dot{\varepsilon}_{11} \neq 0$. Instead of the above derivation, we may take the differential of equation $(2)_{2}$ with respect to $\varepsilon_{11}$ directly. It follows that

$$
\frac{\mathrm{d} \nu_{t}}{\mathrm{~d} \varepsilon_{11}}=\frac{-\varepsilon_{11} \mathrm{~d} \varepsilon_{22} / \mathrm{d} \varepsilon_{11}}{\varepsilon_{11}^{2}}+\frac{\varepsilon_{22}}{\varepsilon_{11}^{2}} .
$$

Noting that $\mathrm{d} \varepsilon_{22} / \mathrm{d} \varepsilon_{11}=-v_{r}$ and $\varepsilon_{22} / \varepsilon_{11}=-v_{t}$ from equation (2) and rearranging the above equation, we obtain equation (4) again.

For isotropic linear elasticity, $v_{r}=v_{t}=v$ is a material constant known as Poisson's ratio, and the bulk modulus is given by

$$
K=\frac{2 G(1+v)}{3(1-2 v)},
$$

where $G$ is the shear modulus. The value $v=1 / 2$ implies $1 / K=0$, i.e., elastic incompressibility.

The axial-torsional control pairs $\left(\sigma_{11}, \sigma_{12}\right),\left(\varepsilon_{11}, \varepsilon_{12}\right),\left(\sigma_{11}, \varepsilon_{12}\right)$, and $\left(\varepsilon_{11}, \sigma_{12}\right)$ are the total components rather than the deviatoric components; therefore, it is important to deal directly with the stress and strain components rather than with their deviatoric parts, even though the volumetric parts are elastic in most metals and hence do not play a dominant role in the study of metal plasticity. Therefore, the constitutive relation of the volumetric parts

$$
\operatorname{tr} \dot{\varepsilon}=\frac{\operatorname{tr} \dot{\sigma}}{3 K}=\frac{\dot{\sigma}_{11}}{3 K}
$$

must always be included in the study. Because tr $\dot{\varepsilon}$ is not yet known, it may greatly complicate the calculation of model responses as will be shown later. Therefore, the values $v_{r}$ and $v_{t}$ were usually taken to be $1 / 2$ in the plastic deformation range for simplicity; see, for example, Khan and Huang (1995). Note that the simplified assumption $v_{r}=v_{t}=1 / 2$ should not be absurdly viewed as a result of plastic incompressibility, a popular assumption for metal plasticity, which means that there is no volumetric plastic deformation in metals.

The rate form contraction ratio was rarely reported in the literature. In this paper we consider the PrandtlReuss model subjected to the above controls and attempt to achieve a deeper understanding of the ratios $v_{r}$ and $v_{t}$ and to assess the validity of the simplified assumption $v_{r}=v_{t}=1 / 2$ prevailing in the plasticity literature. Although our consideration is limited to the Prandtl-Reuss model, the raised issue is considered typical in the models of plasticity, and the method of analysis and solution presented here may apply to some classes of plasticity models more complicated than the Prandtl-Reuss model. 


\section{The model}

The elastoplastic model for solid materials proposed by Prandtl (1924) and Reuss (1930) is re-formulated (see, for example, Hong and Liu (1997)) as follows:

$$
\begin{aligned}
& \dot{\boldsymbol{\varepsilon}}=\dot{\boldsymbol{\varepsilon}}^{e}+\dot{\boldsymbol{\varepsilon}}^{p}, \\
& \dot{\boldsymbol{\sigma}}=2 G \dot{\boldsymbol{\varepsilon}}^{e}+\frac{3 K-2 G}{3}(\operatorname{tr} \dot{\boldsymbol{\varepsilon}}) \mathbf{I}, \\
& \dot{\lambda}\left[\boldsymbol{\sigma}-\frac{1}{3}(\operatorname{tr} \boldsymbol{\sigma}) \mathbf{I}\right]=2 \tau_{y} \dot{\boldsymbol{\varepsilon}}^{p}, \\
& \sqrt{\frac{1}{2}\left[\boldsymbol{\sigma}-\frac{1}{3}(\operatorname{tr} \boldsymbol{\sigma}) \mathbf{I}\right] \cdot\left[\boldsymbol{\sigma}-\frac{1}{3}(\operatorname{tr} \boldsymbol{\sigma}) \mathbf{I}\right]} \leqslant \tau_{y}, \\
& \dot{\lambda} \geqslant 0, \\
& \dot{\lambda} \sqrt{\frac{1}{2}\left[\boldsymbol{\sigma}-\frac{1}{3}(\operatorname{tr} \boldsymbol{\sigma}) \mathbf{I}\right] \cdot\left[\boldsymbol{\sigma}-\frac{1}{3}(\operatorname{tr} \boldsymbol{\sigma}) \mathbf{I}\right]}=\dot{\lambda} \tau_{y},
\end{aligned}
$$

where I is the identity tensor, the symbol tr denotes the trace of the tensor, and a dot between two tensors of the same order denotes their Euclidean inner product. The model has only three experimentally determined material constants, namely the bulk modulus $K$, the shear modulus $G$, and the shear yield strength $\tau_{y}$, which are postulated to be

$$
\frac{1}{K} \geqslant 0, \quad G>0, \quad \tau_{y}>0 .
$$

The boldfaced $\boldsymbol{\varepsilon}, \boldsymbol{\varepsilon}^{e}, \boldsymbol{\varepsilon}^{p}$ and $\sigma$ are, respectively, the strain, elastic strain, plastic strain, and stress tensors, all symmetric, whereas $\lambda$ is a scalar. All the $\varepsilon, \varepsilon^{e}, \boldsymbol{\varepsilon}^{p}, \sigma$ and $\lambda$ are functions of one and the same independent variable, which in most cases is taken either as the ordinary time or as the arc length of the control path; however, for convenience, the independent variable, no matter what it is, will simply be called 'time' and given the symbol $t$. As said earlier, a superimposed dot denotes differentiation with respect to time, $\mathrm{d} / \mathrm{d} t$. Without loss of generality, it is also postulated that with the above differential model, there is a time instant designated as $t_{0}$, called the zero-value (or annealed) time, before and at which the material is in the zero-value (or annealed) state in the sense that the relevant values $\boldsymbol{\varepsilon}\left(t_{0}\right), \boldsymbol{\varepsilon}^{e}\left(t_{0}\right), \boldsymbol{\varepsilon}^{p}\left(t_{0}\right), \boldsymbol{\sigma}\left(t_{0}\right)$ and $\lambda\left(t_{0}\right)$ are all zero. In general the zerovalue time $t_{0}$, the initial time $t_{i}$, and the current time $t$ obey the order $t_{0} \leqslant t_{i} \leqslant t$.

In summary, the material of the thin-walled tube studied in this paper is modelled by equations (6)-(12) and subjected to certain initial conditions at the initial time $t_{i}$.

Taking traces of both sides of equation (8) and noting equation $(12)_{3}$, we have

$$
\operatorname{tr} \dot{\boldsymbol{\varepsilon}}^{p}=0,
$$

that is, the model implies plastic incompressibility. Taking traces of both sides of equation (7) and noting equations (13) and (6) yield the first equality of equation (5), that is, the model implies an elastic relation for 
the volumetric part. The model also implies ${ }^{2}$

$$
E>0, \quad-1<v \leqslant \frac{1}{2}
$$

where

$$
E:=\frac{9 K G}{3 K+G}, \quad v:=\frac{3 K-2 G}{6 K+2 G}
$$

are Young's modulus and Poisson's ratio, respectively. Furthermore, the model validates

$$
\dot{\varepsilon}_{22}=\dot{\varepsilon}_{33}
$$

under the axial-torsional controls; however, neither the model alone nor the axial-torsional controls by themselves can lead to $\dot{\varepsilon}_{22}=\dot{\varepsilon}_{33}$.

In this paper it will be made clear that, for the model under the axial-torsional controls, plastic incompressibility does not lead to $v_{r}=v_{t}=1 / 2$, and the contraction ratios $v_{r}$ and $v_{t}$ and Poisson's ratio $v$ are different from one another.

\section{Axial-torsional problem}

\subsection{Governing equations}

For a two-dimensional axial-torsional problem the following governing equations can be obtained from equations (6)-(8) and (1),

$$
\begin{aligned}
& \frac{\dot{\sigma}_{11}}{\sqrt{3}}+\frac{E}{3} \frac{\dot{\lambda}}{\tau_{y}} \frac{\sigma_{11}}{\sqrt{3}}=\frac{E}{3}\left(\sqrt{3} \dot{\varepsilon}_{11}\right), \\
& \dot{\sigma}_{12}+G \frac{\dot{\lambda}}{\tau_{y}} \sigma_{12}=G\left(2 \dot{\varepsilon}_{12}\right),
\end{aligned}
$$

and the yield condition reduces to

$$
\left(\frac{\sigma_{11}}{\sqrt{3}}\right)^{2}+\sigma_{12}^{2}=\tau_{y}^{2} .
$$

We may use equations (17) and (18) to solve for $\sigma_{11}$ and $\sigma_{12}$, respectively. However, the rate $\dot{\lambda}$ remains to be determined, which is the theme of the next subsection.

\subsection{Switch of plasticity}

In this subsection we shall see that the complementary trios (9)-(11) enable the model to possess an onoff switch of the plastic mechanism, the switching criterion of which will be derived right below. Multiplying

\footnotetext{
${ }^{2}$ That the ranges (14) are the implications of the model can be seen in the following way. Equation (14) $)_{1}$ comes from substituting equations (12) and $(12)_{2}$ in equation $(15)_{1}$, whereas equation $(14)_{2}$ comes from substituting equations $(12)_{1},(12)_{2}$, and (14) 1 in the relations $K=E /(3-6 v)$ and $G=E /(2+2 v)$, which can be obtained by solving equations $(15)_{1}$ and $(15)_{2}$ simultaneously.
} 
equations (17) and (18) by $\sigma_{11} / \sqrt{3}$ and $\sigma_{12}$, respectively, and adding the results lead to

$$
\frac{\sigma_{11} \dot{\sigma}_{11}}{3}+\sigma_{12} \dot{\sigma}_{12}+\frac{E \dot{\lambda}}{3 \tau_{y}}\left(\frac{\sigma_{11}}{\sqrt{3}}\right)^{2}+\frac{G \dot{\lambda}}{\tau_{y}} \sigma_{12}^{2}=\frac{E}{3} \sigma_{11} \dot{\varepsilon}_{11}+2 G \sigma_{12} \dot{\varepsilon}_{12}
$$

Since $\tau_{y}$ is constant, the above equation implies

$$
\sigma_{11}^{2}+3 \sigma_{12}^{2}=3 \tau_{y}^{2} \Rightarrow \dot{\lambda}\left(E \sigma_{11}^{2}+9 G \sigma_{12}^{2}\right)=3 \tau_{y}\left(E \sigma_{11} \dot{\varepsilon}_{11}+6 G \sigma_{12} \dot{\varepsilon}_{12}\right)
$$

Recalling the positivity of $\tau_{y}, E$ and $G$ we have

$$
\sigma_{11}^{2}+3 \sigma_{12}^{2}=3 \tau_{y}^{2} \Rightarrow E \sigma_{11} \dot{\varepsilon}_{11}+6 G \sigma_{12} \dot{\varepsilon}_{12}>0 \Leftrightarrow \dot{\lambda}>0
$$

from which

$$
\left\{\sigma_{11}^{2}+3 \sigma_{12}^{2}=3 \tau_{y}^{2} \text { and } E \sigma_{11} \dot{\varepsilon}_{11}+6 G \sigma_{12} \dot{\varepsilon}_{12}>0\right\} \Rightarrow \dot{\lambda}>0 .
$$

On the other hand, if $\dot{\lambda}>0$, equation (11) ensures $\sigma_{11}^{2}+3 \sigma_{12}^{2}=3 \tau_{y}^{2}$, which together with equation (20) leads to

$$
\dot{\lambda}>0 \Rightarrow\left\{\sigma_{11}^{2}+3 \sigma_{12}^{2}=3 \tau_{y}^{2} \text { and } E \sigma_{11} \dot{\varepsilon}_{11}+6 G \sigma_{12} \dot{\varepsilon}_{12}>0\right\} .
$$

From equations (21) and (22) we thus conclude that the yield condition $\sigma_{11}^{2}+3 \sigma_{12}^{2}=3 \tau_{y}^{2}$ and the straining condition $E \sigma_{11} \dot{\varepsilon}_{11}+6 G \sigma_{12} \dot{\varepsilon}_{12}>0$ are sufficient and necessary for plastic irreversibility $\dot{\lambda}>0$. Considering this and the inequality (10), we thus reveal the following criterion for plastic irreversibility:

$$
\dot{\lambda}= \begin{cases}\frac{3 \tau_{y}\left(E \sigma_{11} \dot{\varepsilon}_{11}+6 G \sigma_{12} \dot{\varepsilon}_{12}\right)}{E \sigma_{11}^{2}+9 G \sigma_{12}^{2}}>0 & \text { if } \sigma_{11}^{2}+3 \sigma_{12}^{2}=3 \tau_{y}^{2} \text { and } E \sigma_{11} \dot{\varepsilon}_{11}+6 G \sigma_{12} \dot{\varepsilon}_{12}>0, \\ 0 & \text { if } \sigma_{11}^{2}+3 \sigma_{12}^{2}<3 \tau_{y}^{2} \text { or } E \sigma_{11} \dot{\varepsilon}_{11}+6 G \sigma_{12} \dot{\varepsilon}_{12} \leqslant 0\end{cases}
$$

According to the complementary trios (9)-(11), there are just two phases: (i) $\dot{\lambda}>0$ and $\sigma_{11}^{2}+3 \sigma_{12}^{2}=3 \tau_{y}^{2}$, and (ii) $\dot{\lambda}=0$ and $\sigma_{11}^{2}+3 \sigma_{12}^{2} \leqslant 3 \tau_{y}^{2}$. From the criterion (23) it is clear that (i) corresponds to the plastic phase (also called the on phase, or the elastoplastic phase) while (ii) to the elastic phase (also called the off phase). During the plastic phase, $\dot{\lambda}>0$, the mechanism of plasticity is on, the mechanical process is irreversible, and the material exhibits elastoplastic behavior, while in the elastic phase, $\dot{\lambda}=0$, the mechanism of plasticity is off, the mechanical process is reversible, and the material responds elastically. Thus equation (23) is called the on-off switching criterion for the on-off switch of the mechanism of plasticity.

\subsection{Difficulties}

Substituting equation (23) for $\dot{\lambda}$ in equations (17) and (18), we obtain

$$
\begin{aligned}
& \dot{\sigma}_{11}+\frac{E\left(E \sigma_{11} \dot{\varepsilon}_{11}+6 G \sigma_{12} \dot{\varepsilon}_{12}\right)}{E \sigma_{11}^{2}+9 G \sigma_{12}^{2}} \sigma_{11}=E \dot{\varepsilon}_{11}, \\
& \dot{\sigma}_{12}+\frac{3 G\left(E \sigma_{11} \dot{\varepsilon}_{11}+6 G \sigma_{12} \dot{\varepsilon}_{12}\right)}{E \sigma_{11}^{2}+9 G \sigma_{12}^{2}} \sigma_{12}=2 G \dot{\varepsilon}_{12} .
\end{aligned}
$$




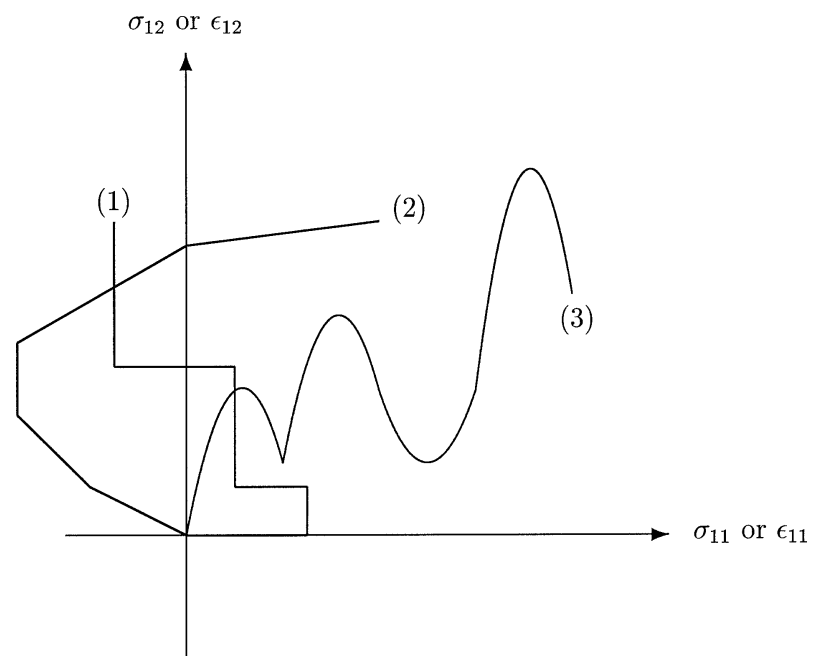

Figure 2. In control path (1) only one component is varied at one time while the other component is held fixed. Path (2) is rectilinear and path (3) is general.

For the strain control and the mixed controls the above two equations are coupled, highly non-linear, and hard to solve $^{3}$, and so it seems desirable to re-examine the conventional strategies for dealing with the axial-torsional problem and the difficulties encountered in their approaches.

Recall that in the process of deriving equation (17) one may as well obtain

$$
\frac{\dot{\sigma}_{11}}{\sqrt{3}}+G \frac{\dot{\lambda}}{\tau_{y}} \frac{\sigma_{11}}{\sqrt{3}}=G \sqrt{3}\left(\dot{\varepsilon}_{11}-\frac{1}{3} \operatorname{tr} \dot{\varepsilon}\right) .
$$

This equation has the same corresponding homogeneous equation as equation (18) does, but contains in the nonhomogeneous term tr $\dot{\varepsilon}$, a quantity not known a priori, which is indeed one part of the responses, depending on the inputs $\dot{\varepsilon}_{11}$ and $\dot{\varepsilon}_{12}$ and the initial stresses. One may measure the hoop strain rate or the radial strain rate or both to obtain tr $\dot{\boldsymbol{\varepsilon}}$. Such measurement is not an easy work but can be taken someway. However, in the model simulation the quantity $\operatorname{tr} \dot{\boldsymbol{\varepsilon}}$ is not an independent variable requiring extra measurement and, therefore, should be calculated through the constitutive laws. Accordingly, using equation (5) in equation (26), one obtains equation (17) again, and so one still encounters the difficulty of solving the highly non-linear equations (24) and (25). Generally speaking, this difficulty was treated in the literature of plasticity by two approaches aside from the measurement. The first approach was to restrict the paths by moving only one of the two components at one time, while holding the other component fixed, as illustrated by path (1) in figure 2 . Then the problem can be seen to be exactly solvable. The second approach was to simply neglect the term tr $\dot{\boldsymbol{\varepsilon}}$ with various excuses for the neglecting. Under the model of equations (6)-(12) the following excuses are equivalent and any one of them can lead correctly to $\operatorname{tr} \dot{\boldsymbol{\varepsilon}}=0$ : (i) elastic incompressibility (or $v=1 / 2$ or $1 / K=0$ or $E=3 G$ ), (ii) incompressibility. Be cautious that some excuses given in the literature were too restricted to be consistent with the whole problem. However, the second approach still results in non-linear differential equations, and so in the next subsection we shall analyse the second approach in a new perspective, and then in the next section devise a new formulation to unlock the difficulty.

\footnotetext{
${ }^{3}$ For the pure stress control equations (24) and (25) are linear and easy to solve, but, in reality, in the axial-torsional testing, the pure stress control is a risky job, often leading to unstable deformation and abrupt collapse of the specimen.
} 
It cannot be overemphasized that we have to face the difficulty arising from elastic compressibility and to develop a new formulation if we wish to investigate the theoretical variations of the contraction ratios $v_{r}$ and $v_{t}$; otherwise, should we assume elastic incompressibility, we would obtain the trivial answers $v=v_{r}=1 / 2$ and, if $\operatorname{tr} \boldsymbol{\varepsilon}\left(t_{i}\right)=0, v_{t}=1 / 2$.

\subsection{The incompressible model}

Under the additional assumption of elastic incompressibility (or $v=1 / 2$ or $1 / K=0$ or $E=3 G$ ), the on-off switching criterion (23) of the model (6)-(12) reduces to

$$
\dot{\lambda}= \begin{cases}\frac{1}{\tau_{y}}\left(\sigma_{11} \dot{\varepsilon}_{11}+2 \sigma_{12} \dot{\varepsilon}_{12}\right)>0 & \text { if } \sigma_{11}^{2}+3 \sigma_{12}^{2}=3 \tau_{y}^{2} \text { and } \sigma_{11} \dot{\varepsilon}_{11}+2 \sigma_{12} \dot{\varepsilon}_{12}>0, \\ 0 & \text { if } \sigma_{11}^{2}+3 \sigma_{12}^{2}<3 \tau_{y}^{2} \text { or } \sigma_{11} \dot{\varepsilon}_{11}+2 \sigma_{12} \dot{\varepsilon}_{12} \leqslant 0\end{cases}
$$

and equations (26) and (18) reduce to

$$
\begin{aligned}
& \frac{1}{\tau_{y}}\left(\frac{\dot{\sigma}_{11}}{\sqrt{3}}+\frac{\dot{X}^{0}}{X^{0}} \frac{\sigma_{11}}{\sqrt{3}}\right)=\frac{\sqrt{3} G}{\tau_{y}} \dot{\varepsilon}_{11}, \\
& \frac{1}{\tau_{y}}\left(\dot{\sigma}_{12}+\frac{\dot{X}^{0}}{X^{0}} \sigma_{12}\right)=\frac{2 G}{\tau_{y}} \dot{\varepsilon}_{12},
\end{aligned}
$$

where

$$
X^{0}(t):=\exp \frac{G \lambda(t)}{\tau_{y}}
$$

is the integrating factor. Concentrating on the plastic phase, we re-write equation $(27)_{1}$ as

$$
\frac{\dot{X}^{0}}{X^{0}}=\frac{G}{\tau_{y}^{2}}\left(\sigma_{11} \dot{\varepsilon}_{11}+2 \sigma_{12} \dot{\varepsilon}_{12}\right)
$$

As a consequence, for the plastic phase equations (28), (29), and (30) can be arranged in the matrix form:

$$
\frac{\mathrm{d}}{\mathrm{d} t}\left[\begin{array}{c}
X^{0} \frac{\sigma_{11}}{\sqrt{3} \tau_{y}} \\
X^{0} \frac{\sigma_{12}}{\tau_{y}} \\
X^{0}
\end{array}\right]=\frac{G}{\tau_{y}}\left[\begin{array}{ccc}
0 & 0 & \sqrt{3} \dot{\varepsilon}_{11} \\
0 & 0 & 2 \dot{\varepsilon}_{12} \\
\sqrt{3} \dot{\varepsilon}_{11} & 2 \dot{\varepsilon}_{12} & 0
\end{array}\right]\left[\begin{array}{c}
X^{0} \frac{\sigma_{11}}{\sqrt{3} \tau_{y}} \\
X^{0} \frac{\sigma_{12}}{\tau_{y}} \\
X^{0}
\end{array}\right] .
$$

This is a system of linear equations for the so-called augmented stress vector (Hong and Liu, 2000)

$$
\mathbf{X}:=\left[\begin{array}{c}
X^{0} \frac{\sigma_{11}}{\sqrt{3} \tau_{y}} \\
X^{0} \frac{\sigma_{12}}{\tau_{y}} \\
X^{0}
\end{array}\right]
$$

and the control tensor

$$
\mathbf{A}:=\frac{G}{\tau_{y}}\left[\begin{array}{ccc}
0 & 0 & \sqrt{3} \dot{\varepsilon}_{11} \\
0 & 0 & 2 \dot{\varepsilon}_{12} \\
\sqrt{3} \dot{\varepsilon}_{11} & 2 \dot{\varepsilon}_{12} & 0
\end{array}\right]
$$


is really an element of the Lie algebra of the proper orthochronous Lorentz group $\mathrm{SO}_{0}(2,1)$, namely $\mathbf{A} \in$ so $(2,1)$. From the formulation above it is easy to obtain the closed-form solutions of the responses under rectilinear strain paths; see, for example, Hong and Liu (1998). However, this scheme does not directly apply to equations (26) and (18) when tr $\dot{\varepsilon}$ does not vanish due to elastic compressibility. Instead of the above exact linearization technique a new formulation will be developed in the next section to tackle this difficulty arising from the non-vanishing of $\operatorname{tr} \dot{\boldsymbol{\varepsilon}}$.

\section{A new formulation for simulation}

In this section we concentrate our investigation on the plastic phase for general axial-torsional control paths, as illustrated by path (3) in figure 2 .

\subsection{Change of variables}

Let

$$
\mathbf{Q}(t):=\left[\begin{array}{c}
\frac{\sigma_{11}(t)}{\sqrt{3} \tau_{y}} \\
\frac{\sigma_{12}(t)}{\tau_{y}}
\end{array}\right], \quad \dot{\mathbf{q}}(t):=\left[\begin{array}{c}
\sqrt{3}\left[\dot{\varepsilon}_{11}(t)-\frac{1}{3} \operatorname{tr} \dot{\boldsymbol{\varepsilon}}(t)\right] \\
2 \dot{\varepsilon}_{12}(t)
\end{array}\right], \quad \mathbf{Q}^{\perp}(t):=\left[\begin{array}{c}
-\frac{\sigma_{12}(t)}{\tau_{y}} \\
\sigma_{11}(t) \\
\sqrt{3} \tau_{y}
\end{array}\right] .
$$

Then equations (26) and (18) can be recast to

$$
\dot{\mathbf{Q}}+\frac{\dot{\lambda}}{q_{y}} \mathbf{Q}=\frac{\dot{\mathbf{q}}}{q_{y}},
$$

where $q_{y}:=\tau_{y} / G$ is the shear yield strain, and the yield condition (19) becomes

$$
\|\mathbf{Q}\|=1,
$$

where $\|\mathbf{Q}\|:=\sqrt{\mathbf{Q} \cdot \mathbf{Q}}$. It is easy to see that

$$
\|\mathbf{Q}\|=1, \quad\left\|\mathbf{Q}^{\perp}\right\|=1, \quad \mathbf{Q}^{\perp} \cdot \mathbf{Q}=0
$$

for all $t \geqslant t_{i}$ in a plastic-phase time interval. Thus the two constant orthonormal vectors $\mathbf{Q}\left(t_{i}\right)$ and $\mathbf{Q}^{\perp}\left(t_{i}\right)$ span a two-dimensional space, which lie in all stress paths for the axial-torsional problem.

Since $\mathbf{Q}(t), \dot{\mathbf{q}}(t)$, and $\mathbf{Q}^{\perp}\left(t_{i}\right)$ are two-dimensional vectors, two scalars $\dot{x}(t)$ and $\dot{u}(t)$ suffice to ensure

$$
\frac{\dot{\mathbf{q}}(t)}{q_{y}}=\dot{u}(t) \mathbf{Q}(t)+\dot{x}(t) \mathbf{Q}^{\perp}\left(t_{i}\right)
$$

to hold for $t \geqslant t_{i}$ until $\mathbf{Q}$ rotates 90 degrees so as to coincide with $\mathbf{Q}^{\perp}\left(t_{i}\right)$. Substituting equation (36) into equation (33) we obtain

$$
\dot{\mathbf{Q}}+\frac{\dot{y}}{y} \mathbf{Q}=\dot{x} \mathbf{Q}^{\perp}\left(t_{i}\right)
$$

where

$$
\frac{\dot{y}}{y}:=\frac{\dot{\lambda}}{q_{y}}-\dot{u} .
$$


Integrating equation (37) yields

$$
\mathbf{Q}(t)=\frac{y\left(t_{i}\right)}{y(t)} \mathbf{Q}\left(t_{i}\right)+\mathbf{Q}^{\perp}\left(t_{i}\right) \int_{t_{i}}^{t} \frac{y(\xi)}{y(t)} \dot{x}(\xi) \mathrm{d} \xi
$$

To summarize, we have made in this subsection a change of variables from $\sigma_{11}$ (or $\varepsilon_{11}$ ), $\sigma_{12}$ (or $\varepsilon_{12}$ ), $\dot{\lambda}$, tr $\dot{\boldsymbol{\varepsilon}}$ in equations (26) and (18) to $\sigma_{11}, \sigma_{12}, x, y$ in equation (39). This change will soon prove to be rewarding.

\subsection{Stress in terms of $x$}

Due to $\mathbf{Q} \cdot \mathbf{Q}=1$ the inner product of equation (37) with $\mathbf{Q}$ is

$$
\frac{\dot{y}}{y}=\dot{x} \mathbf{Q}^{\perp}\left(t_{i}\right) \cdot \mathbf{Q}
$$

Substituting equation (39) for $\mathbf{Q}$ in the above equation, using equation (35), and recalling $\dot{x} \neq 0$, we obtain

$$
y^{\prime}(x(t))=\int_{x\left(t_{i}\right)}^{x(t)} y(x(\xi)) \mathrm{d} x(\xi),
$$

where the prime denotes differentiation with respect to $x$. Equation (41) is a linear Volterra integrodifferential equation for $y(x)$. Differentiating equation (41) with respect to $x$ yields

$$
y^{\prime \prime}=y \text {. }
$$

If we assign $x\left(t_{i}\right)=0$ and $y\left(t_{i}\right)=1$, then we readily find that

$$
y(x(t))=\cosh x(t)
$$

where $y^{\prime}\left(x\left(t_{i}\right)\right)=0$ due to equation (41) has been used, and the assignment is legitimate because it is $\dot{x}$ and $\dot{y} / y$ rather than $x$ and $y$ that were introduced earlier.

Define

$$
\begin{gathered}
\mathbf{F}=\left[\begin{array}{c}
F_{1} \\
F_{2}
\end{array}\right]:=\mathbf{Q}\left(t_{i}\right)+(\sinh x) \mathbf{Q}^{\perp}\left(t_{i}\right)=\left[\begin{array}{c}
\frac{\sigma_{11}\left(t_{i}\right)}{\sqrt{3} \tau_{y}}-\frac{\sigma_{12}\left(t_{i}\right)}{\tau_{y}} \sinh x \\
\frac{\sigma_{12}\left(t_{i}\right)}{\tau_{y}}+\frac{\sigma_{11}\left(t_{i}\right)}{\sqrt{3} \tau_{y}} \sinh x
\end{array}\right], \\
\mathbf{F}^{\perp}:=\left[\begin{array}{c}
-F_{2} \\
F_{1}
\end{array}\right]=(-\sinh x) \mathbf{Q}\left(t_{i}\right)+\mathbf{Q}^{\perp}\left(t_{i}\right)=\left[\begin{array}{c}
-\frac{\sigma_{12}\left(t_{i}\right)}{\tau_{y}}-\frac{\sigma_{11}\left(t_{i}\right)}{\sqrt{3} \tau_{y}} \sinh x \\
\frac{\sigma_{11}\left(t_{i}\right)}{\sqrt{3} \tau_{y}}-\frac{\sigma_{12}\left(t_{i}\right)}{\tau_{y}} \sinh x
\end{array}\right] .
\end{gathered}
$$

Note that $\mathbf{F}=\mathbf{F}\left(x(t), \sigma_{11}\left(t_{i}\right), \sigma_{22}\left(t_{i}\right)\right), \mathbf{F}^{\perp}=\mathbf{F}^{\perp}\left(x(t), \sigma_{11}\left(t_{i}\right), \sigma_{22}\left(t_{i}\right)\right)$, and $\mathbf{F} \cdot \mathbf{F}^{\perp}=0$. With the aid of $y=$ $\cosh x, y\left(t_{i}\right)=1$, and definition (44), equation (39) reduces to

$$
\mathbf{Q}(t)=\frac{\mathbf{F}}{\cosh x} .
$$




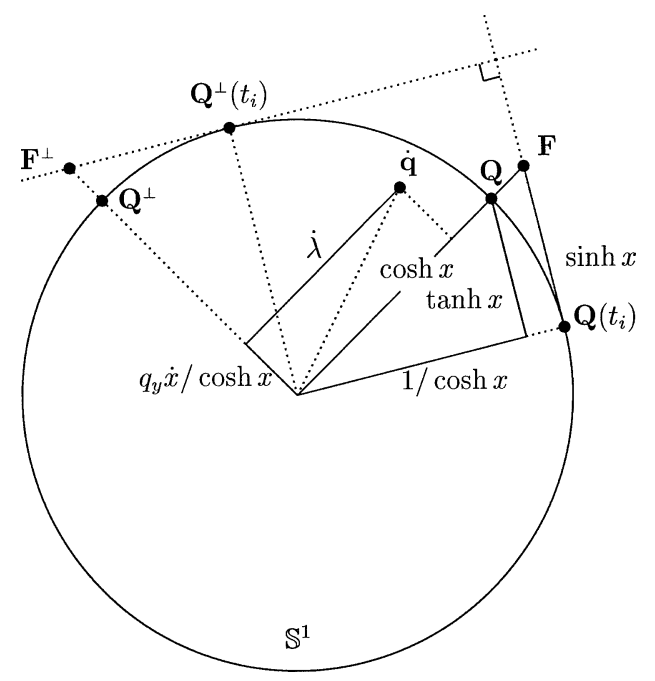

Figure 3. In the normalized stress plane the yield locus becomes a unit circle $\mathbb{S}^{1}$. The normalized stress $\mathbf{Q}$ is decomposed into $(1 / \cosh x) \mathbf{Q}\left(t_{i}\right)$ and $(\tanh x) \mathbf{Q}^{\perp}\left(t_{i}\right)$, whereas the normalized strain rate $\dot{\mathbf{q}}$ is decomposed into $\dot{\lambda} \mathbf{Q}$ and $\left(q_{y} \dot{x} / \cosh x\right) \mathbf{Q}^{\perp}$.

Equation (46) indicates that the normalized stress vector $\mathbf{Q}(t)$ always lies in the space spanned by the orthonormal vectors $\mathbf{Q}\left(t_{i}\right)$ and $\mathbf{Q}^{\perp}\left(t_{i}\right)$, which is two-dimensional and may therefore be called the normalized stress plane of the axial-torsional problem; see figure 3 for such decomposition. It is worth double checking $\|\mathbf{Q}(t)\|=\left\|\mathbf{Q}\left(t_{i}\right)\right\|=1$ using the expressions in equations (46) and (44); hence, the method presented here preserves the so-called consistency condition automatically.

\subsection{Time history of $x$}

For the strain control ${ }^{4}$, before calculating $\mathbf{Q}$ we need one more equation to solve for $x$. This can be done as follows. Substituting equation (46) into equation (36) yields

$$
\frac{\dot{\mathbf{q}}}{q_{y}}=\frac{\dot{u}}{\cosh x} \mathbf{F}+\dot{x} \mathbf{Q}^{\perp}\left(t_{i}\right) .
$$

Taking the inner products of equation (47) with $\mathbf{Q}\left(t_{i}\right) \sinh x$ and with $\mathbf{Q}^{\perp}\left(t_{i}\right)$, subtracting the resulting two equations, and recalling equation (35) and definition (45), we obtain

$$
\dot{x}=\frac{\dot{\mathbf{q}}}{q_{y}} \cdot \mathbf{F}^{\perp} .
$$

Taking the time derivative of equation (46) we have

$$
\dot{\mathbf{Q}}=-\frac{\dot{x} \sinh x}{\cosh ^{2} x} \mathbf{F}+\dot{x} \mathbf{Q}^{\perp}\left(t_{i}\right)
$$

\footnotetext{
${ }^{4}$ See comment 5 in subsection 4.5 for the other controls.
} 
Using the first component of the above equation in $\operatorname{tr} \dot{\varepsilon}=\dot{\sigma}_{11} /(3 K)$ and recalling equations (32) and (44), we have

$$
\operatorname{tr} \dot{\boldsymbol{\varepsilon}}=-\frac{\sqrt{3} \tau_{y}}{3 K} \frac{\dot{x}}{\cosh ^{2} x} F_{2}
$$

by which equation (48) becomes

$$
\dot{x}=\frac{3 K G \cosh ^{2} x\left(2 \dot{\varepsilon}_{12} F_{1}-\sqrt{3} \dot{\varepsilon}_{11} F_{2}\right)}{\tau_{y}\left(3 K \cosh ^{2} x+G F_{2}^{2}\right)} .
$$

Equation (51) and the two components of equation (46) can be written out as follows:

$$
\begin{aligned}
\dot{x}= & \frac{6 K G \cosh ^{2} x\left[\frac{\sigma_{11}\left(t_{i}\right)}{\sqrt{3}}-\sigma_{12}\left(t_{i}\right) \sinh x\right]}{3 K \tau_{y}^{2} \cosh ^{2} x+G\left[\sigma_{12}\left(t_{i}\right)+\frac{\sigma_{11}\left(t_{i}\right)}{\sqrt{3}} \sinh x\right]^{2}} \dot{\varepsilon}_{12} \\
& -\frac{3 \sqrt{3} K G \cosh ^{2} x\left[\sigma_{12}\left(t_{i}\right)+\frac{\sigma_{11}\left(t_{i}\right)}{\sqrt{3}} \sinh x\right]}{3 K \tau_{y}^{2} \cosh ^{2} x+G\left[\sigma_{12}\left(t_{i}\right)+\frac{\sigma_{11}\left(t_{i}\right)}{\sqrt{3}} \sinh x\right]^{2}} \dot{\varepsilon}_{11}, \\
\sigma_{11}(t)= & \frac{\sigma_{11}\left(t_{i}\right)-\sqrt{3} \sigma_{12}\left(t_{i}\right) \sinh x}{\cosh x} \\
\sigma_{12}(t)= & \frac{\sigma_{12}\left(t_{i}\right)+\frac{\sigma_{11}\left(t_{i}\right)}{\sqrt{3}} \sinh x}{\cosh x} .
\end{aligned}
$$

These equations apply to general axial-torsional strain control inputs like path (3) in figure 2. Thus we have succeeded in transforming the highly non-linear coupled equations (24) and (25) to a single first-order differential equation (52), the integral of which can give the time history of $x$, which can then be plugged into formulae (53) and (54) to calculate the response histories of the axial stress $\sigma_{11}$ and the shear stress $\sigma_{12}$, respectively. Finally, the hoop strain and the radial strain can be evaluated by

$$
\begin{aligned}
& \varepsilon_{22}(t)=\varepsilon_{22}\left(t_{i}\right)+\frac{1}{2}\left[\varepsilon_{11}\left(t_{i}\right)-\varepsilon_{11}(t)\right]+\frac{\sigma_{11}(t)-\sigma_{11}\left(t_{i}\right)}{6 K}, \\
& \varepsilon_{33}(t)=\varepsilon_{33}\left(t_{i}\right)+\frac{1}{2}\left[\varepsilon_{11}\left(t_{i}\right)-\varepsilon_{11}(t)\right]+\frac{\sigma_{11}(t)-\sigma_{11}\left(t_{i}\right)}{6 K},
\end{aligned}
$$

respectively. These two formulae have been derived from equations (5) and (16). Note that although $\dot{\varepsilon}_{22}(t)=$ $\dot{\varepsilon}_{33}(t)$ for the specimens made of the Prandtl-Reuss material during the axial-torsional testing, it is not unusual that manufacturing and handling before testing may cause $\varepsilon_{22}\left(t_{i}\right) \neq \varepsilon_{33}\left(t_{i}\right)$ in the specimens.

\subsection{Dissipation}

From equations (8), (11), (13), and (23) $)_{1}$, we can calculate the specific power of dissipation, namely the dissipated energy per unit time per unit volume, by

$$
\boldsymbol{\sigma} \cdot \dot{\boldsymbol{\varepsilon}}^{p}=\tau_{y} \dot{\lambda}=\frac{3 \tau_{y}^{2}\left(E \sigma_{11} \dot{\varepsilon}_{11}+6 G \sigma_{12} \dot{\varepsilon}_{12}\right)}{E \sigma_{11}^{2}+9 G \sigma_{12}^{2}}
$$


in the plastic phase. In the elastic phase it is simply zero. Taking the inner product of equation (33) with $\mathbf{Q}$ and using $\|\mathbf{Q}\|=1$ we obtain

$$
\dot{\lambda}=\mathbf{Q} \cdot \dot{\mathbf{q}} \text {. }
$$

Obviously, it is the pair $\left(\sigma, \dot{\boldsymbol{\varepsilon}}^{p}\right)$, or $\left(\tau_{y}, \dot{\lambda}\right)$, or $\left(\tau_{y} \mathbf{Q}, \dot{\mathbf{q}}\right)$, not the pair $(\boldsymbol{\sigma}, \dot{\boldsymbol{\varepsilon}})$, that constitutes a dissipation-power conjugacy. Figure 3 provides a geometric interpretation of equations (48) and (58).

\subsection{Comments on the new formulation}

Some comments on the new formulation are in order.

Comment 1. From equations (53) and (54) it follows that

$$
\sigma_{11}^{2}(t)+3 \sigma_{12}^{2}(t)=\sigma_{11}^{2}\left(t_{i}\right)+3 \sigma_{12}^{2}\left(t_{i}\right)=3 \tau_{y}^{2} .
$$

Thus we can view equations (53) and (54) as a parametric representation of the responses, which match the initial conditions and satisfy the consistency condition automatically.

Comment 2. We may start directly from equation (46) by substituting equations (53) and (54) into equation (23) 1 to get $\dot{\lambda}$, and then substituting $\dot{\lambda}$ and equation (53) into equation (17) or substituting $\dot{\lambda}$ and equation (54) into equation (18) to get the governing equation of $x$. However, these procedures lead to the same result as given by equation (52).

Comment 3. The variables $u$ and $y$ introduced in equations (36) and (38) do not appear in the final equations (52), (53), and (54); however, they may help us understand the derivation of the new formulation.

Comment 4. Equations (37) and (40) can be rearranged in the matrix form:

$$
\frac{\mathrm{d}}{\mathrm{d} x}\left[\begin{array}{c}
y \frac{\sigma_{11}}{\sqrt{3} \tau_{y}} \\
y \frac{\sigma_{12}}{\tau_{y}} \\
y
\end{array}\right]=\frac{1}{\tau_{y}}\left[\begin{array}{ccc}
0 & 0 & -\sigma_{12}\left(t_{i}\right) \\
0 & 0 & \sigma_{11}\left(t_{i}\right) / \sqrt{3} \\
-\sigma_{12}\left(t_{i}\right) & \sigma_{11}\left(t_{i}\right) / \sqrt{3} & 0
\end{array}\right]\left[\begin{array}{c}
y \frac{\sigma_{11}}{\sqrt{3} \tau_{y}} \\
y \frac{\sigma_{12}}{\tau_{y}} \\
y
\end{array}\right] .
$$

Observe that the structure of the above equation is similar to that of equation (31). Both possess the structures of the Lie algebras $\mathrm{so}(2,1)$ of the proper orthochronous Lorentz groups $\mathrm{SO}_{0}(2,1)$, but the Lorentz group of the above equation has $x$ as its group parameter, which in turn evolves with $t$ in a complicated way as indicated in equation (52), whereas the Lorentz group of equation (31) has $t$ directly as the group parameter.

Comment 5. For the pure stress control we can directly solve equations (24) and (25) without any difficulty. For the mixed controls, by using equation (53) for the control $\left(\sigma_{11}, \varepsilon_{12}\right)$, or equation (54) for $\left(\varepsilon_{11}, \sigma_{12}\right)$, we can directly obtain the time history of $x$ and then via $x(t)$ readily calculate all other responses, and so for the mixed controls we are free of solving the first order differential equation (52).

Comment 6. The methodology developed in the new formulation allows us to easily extend it to solve the axial-torsional problems of more complicated constitutive models, which render $\dot{\mathbf{q}}$ more complicated than that defined in equation $(32)_{2}$. Substituting such $\dot{\mathbf{q}}$ into equation (48) will still result in a first order governing equation of $x$. Thus the responses can be calculated similarly. 


\section{Limit strength and discontinuity of contraction ratio}

In this section we consider rectilinear strain paths, like path (2) in figure 2, specified by non-zero constant two-dimensional vectors $\left(\dot{\varepsilon}_{11}, \dot{\varepsilon}_{12}\right)$, namely

$$
\varepsilon_{11}(t)=\varepsilon_{11}\left(t_{i}\right)+\left(t-t_{i}\right) \dot{\varepsilon}_{11}, \quad \varepsilon_{12}(t)=\varepsilon_{12}\left(t_{i}\right)+\left(t-t_{i}\right) \dot{\varepsilon}_{12}
$$

with

$$
\dot{\varepsilon}_{11}=\text { constant }_{1}, \quad \dot{\varepsilon}_{12}=\text { constant }_{2}
$$

being not both zeros. The paths are simple but more general than, for example, path (1) in figure 2 and are frequently encountered in experimental tests and numerical calculations. The initial strain vector $\left(\varepsilon_{11}\left(t_{i}\right), \varepsilon_{12}\left(t_{i}\right)\right)$ has no effect on responses, while, to the contrary, the initial stress vector $\left(\sigma_{11}\left(t_{i}\right), \sigma_{12}\left(t_{i}\right)\right)$ does have significant effects on responses.

\subsection{Switch-on time}

Given the strain path (59) and an initial stress point $\left(\sigma_{11}\left(t_{i}\right), \sigma_{12}\left(t_{i}\right)\right)$ which is confined by the inequality $\sigma_{11}^{2}\left(t_{i}\right)+3 \sigma_{12}^{2}\left(t_{i}\right) \leqslant 3 \tau_{y}^{2}$, if at the initial time $t_{i}$ the mechanism of plasticity is not yet switched on, we want to predict the switch-on time $t_{\mathrm{on}}$ at which it will be turned on. The switch-on time can be determined according to the criterion (23) as follows. First solve for $t$ the following algebraic equation

$$
\left\{\sigma_{11}\left(t_{i}\right)+E\left[\varepsilon_{11}(t)-\varepsilon_{11}\left(t_{i}\right)\right]\right\}^{2}+3\left\{\sigma_{12}\left(t_{i}\right)+2 G\left[\varepsilon_{12}(t)-\varepsilon_{12}\left(t_{i}\right)\right]\right\}^{2}=3 \tau_{y}^{2},
$$

which has been obtained by substituting the elastic equations:

$$
\sigma_{11}(t)=\sigma_{11}\left(t_{i}\right)+E\left[\varepsilon_{11}(t)-\varepsilon_{11}\left(t_{i}\right)\right], \quad \sigma_{12}(t)=\sigma_{12}\left(t_{i}\right)+2 G\left[\varepsilon_{12}(t)-\varepsilon_{12}\left(t_{i}\right)\right]
$$

into the yield condition $\sigma_{11}^{2}(t)+3 \sigma_{12}^{2}(t)=3 \tau_{y}^{2}$. However, the solution $t$ of equation (61) must satisfy $E \sigma_{11}(t) \dot{\varepsilon}_{11}+6 G \sigma_{12}(t) \dot{\varepsilon}_{12}>0$ in order to be a switch-on time $t_{\mathrm{on}}$. If no solution exists to equation (61) or the solution $t$ to equation (61) does not satisfy $E \sigma_{11}(t) \dot{\varepsilon}_{11}+6 G \sigma_{12}(t) \dot{\varepsilon}_{12}>0$, then the strain path will not switch on the plastic mechanism.

Substituting equation (59) into equation (61) we obtain a quadratic equation for $t-t_{i}$,

$$
A\left(t-t_{i}\right)^{2}+B\left(t-t_{i}\right)+C=0
$$

where

$$
A:=E^{2} \dot{\varepsilon}_{11}^{2}+12 G^{2} \dot{\varepsilon}_{12}^{2}, \quad B:=2 E \sigma_{11}\left(t_{i}\right) \dot{\varepsilon}_{11}+12 G \sigma_{12}\left(t_{i}\right) \dot{\varepsilon}_{12}, \quad C:=\sigma_{11}^{2}\left(t_{i}\right)+3 \sigma_{12}^{2}\left(t_{i}\right)-3 \tau_{y}^{2} .
$$

Thus, we have

$$
t_{\mathrm{on}}= \begin{cases}t_{i} & \text { if } C=0 \text { and } B \geqslant 0, \\ t_{i}-\frac{B}{A} & \text { if } C=0 \text { and } B<0, \\ t_{i}+\frac{-B+\sqrt{B^{2}-4 A C}}{2 A} & \text { if } C<0 .\end{cases}
$$




\subsection{Exact solution of $x$}

Under the strain path (59), equation (52) can be written as

$$
\frac{\left(a_{0}+a_{1} \sinh x+a_{2} \sinh ^{2} x\right) \mathrm{d} x}{\left(1+\sinh ^{2} x\right)\left(b_{0}-b_{1} \sinh x\right)}=\mathrm{d} t
$$

where

$$
\begin{aligned}
& a_{0}:=G \sigma_{12}^{2}\left(t_{i}\right)+3 K \tau_{y}^{2}, \quad a_{1}:=\frac{2}{\sqrt{3}} G \sigma_{11}\left(t_{i}\right) \sigma_{12}\left(t_{i}\right), \quad a_{2}:=\frac{G}{3} \sigma_{11}^{2}\left(t_{i}\right)+3 K \tau_{y}^{2}, \\
& b_{0}:=\sqrt{3} G K\left[2 \sigma_{11}\left(t_{i}\right) \dot{\varepsilon}_{12}-3 \sigma_{12}\left(t_{i}\right) \dot{\varepsilon}_{11}\right], \quad b_{1}:=3 G K\left[\sigma_{11}\left(t_{i}\right) \dot{\varepsilon}_{11}+2 \sigma_{12}\left(t_{i}\right) \dot{\varepsilon}_{12}\right] .
\end{aligned}
$$

The left-hand side of equation (63) can be decomposed into

$$
\frac{\left(a_{0}+a_{1} \sinh x+a_{2} \sinh ^{2} x\right) \mathrm{d} x}{\left(1+\sinh ^{2} x\right)\left(b_{0}-b_{1} \sinh x\right)}=\frac{\left(c_{0}+c_{1} \sinh x\right) \mathrm{d} x}{1+\sinh ^{2} x}+\frac{c_{2} \mathrm{~d} x}{b_{0}-b_{1} \sinh x},
$$

where

$$
c_{0}:=\frac{b_{0}\left(a_{0}-a_{2}\right)-a_{1} b_{1}}{b_{0}^{2}+b_{1}^{2}}, \quad c_{1}:=\frac{b_{1}\left(a_{0}-a_{2}\right)+a_{1} b_{0}}{b_{0}^{2}+b_{1}^{2}}, \quad c_{2}:=a_{2}+b_{1} c_{1} .
$$

Substituting equation (64) into equation (63) then integrating the left-hand side from 0 to $x$ and the right-hand side from $t_{i}$ to $t$, we obtain

$$
c_{0} \tanh x+c_{1}-\frac{c_{1}}{\cosh x}+\frac{2 c_{2}}{\sqrt{b_{0}^{2}+b_{1}^{2}}}\left[\operatorname{arth}\left(\frac{b_{1}+b_{0} \tanh x / 2}{\sqrt{b_{0}^{2}+b_{1}^{2}}}\right)-\operatorname{arth}\left(\frac{b_{1}}{\sqrt{b_{0}^{2}+b_{1}^{2}}}\right)\right]=t-t_{i},
$$

arth denoting the hyperbolic arctangent function. This equation provides the exact solution of $x$ in an implicit function form.

\subsection{Limit strength}

In this subsection we study the limit states, especially the limit (or residual) strengths and the limit dissipation powers, under rectilinear strain paths. In the stress plane $\left(\sigma_{11}, \sigma_{12}\right)$, the limit strength vector $\left(\bar{\sigma}_{11}, \bar{\sigma}_{12}\right)$ is indeed the fixed point of the dynamical system of equations (24) and (25), and, therefore, can be obtained by letting $\dot{\sigma}_{11}=\dot{\sigma}_{22}=0$ in equations (24) and (25) and solving the resultant equations. Accordingly,

$$
\bar{\sigma}_{11}=\frac{3 \tau_{y} \dot{\varepsilon}_{11}}{\sqrt{3 \dot{\varepsilon}_{11}^{2}+4 \dot{\varepsilon}_{12}^{2}}}, \quad \bar{\sigma}_{12}=\frac{2 \tau_{y} \dot{\varepsilon}_{12}}{\sqrt{3 \dot{\varepsilon}_{11}^{2}+4 \dot{\varepsilon}_{12}^{2}}} .
$$

The corresponding limit value $\bar{x}$ of $x$ can be obtained by letting $\dot{x}=0$ in equation (52) and solving the resultant equation; thus

$$
\bar{x}=\operatorname{arsh} \frac{2 \sigma_{11}\left(t_{i}\right) \dot{\varepsilon}_{12}-3 \sigma_{12}\left(t_{i}\right) \dot{\varepsilon}_{11}}{\sqrt{3}\left[\sigma_{11}\left(t_{i}\right) \dot{\varepsilon}_{11}+2 \sigma_{12}\left(t_{i}\right) \dot{\varepsilon}_{12}\right]},
$$

arsh denoting the hyperbolic arcsine function. Note that $\bar{x}$ is an invariant of equation (52), since substituting it into equations (53) and (54) leads to equation (66) again. Substituting equation (66) into (23) 1 we obtain the 
limit dissipation power

$$
\tau_{y} \overline{\dot{\lambda}}=\tau_{y} \sqrt{3 \dot{\varepsilon}_{11}^{2}+4 \dot{\varepsilon}_{12}^{2}}
$$

Figure 4 shows four examples of the responses $\left(\sigma_{11}, \sigma_{12}, \dot{\varepsilon}_{22}, \dot{\lambda}\right)$ to the same input strain paths but with different initial stress points; the same input strain paths were rectilinear with constant strain rate vector $\left(\dot{\varepsilon}_{11}, \dot{\varepsilon}_{12}\right)=(0.002,-0.001) \mathrm{s}^{-1}$ and the four different initial stress points $\left(\sigma_{11}\left(t_{i}\right), \sigma_{12}\left(t_{i}\right)\right)$ were

(a)

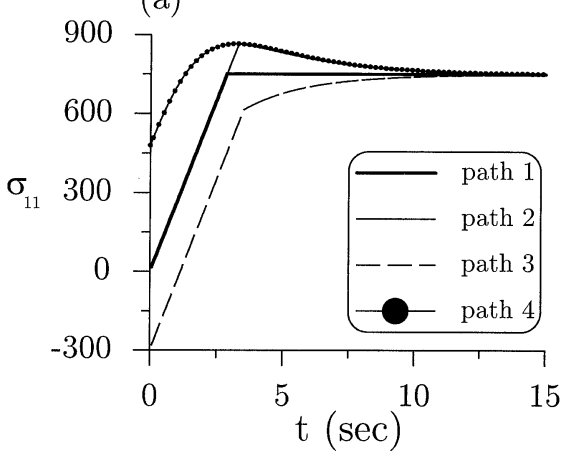

(b)
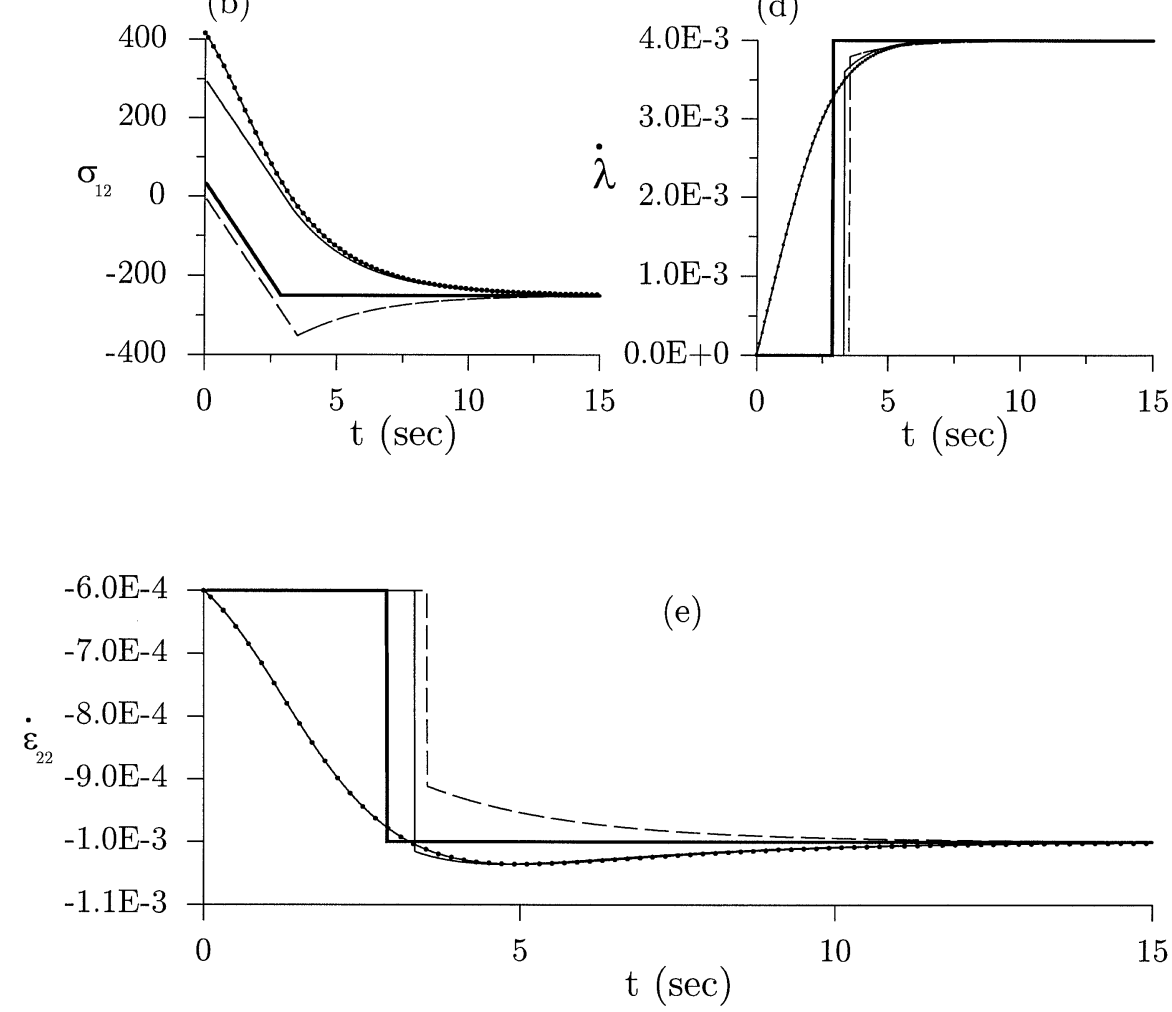

(c)

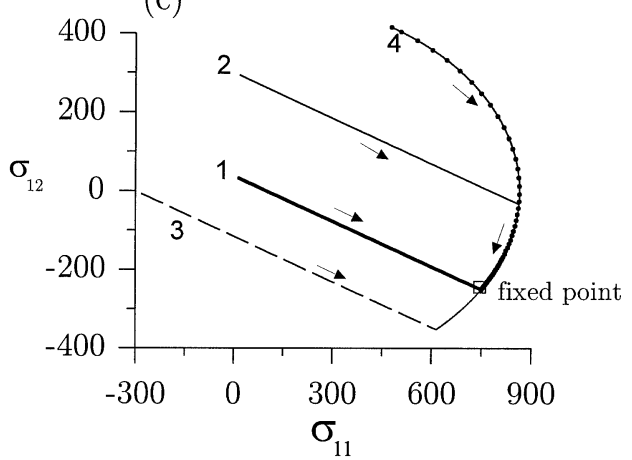

(d)

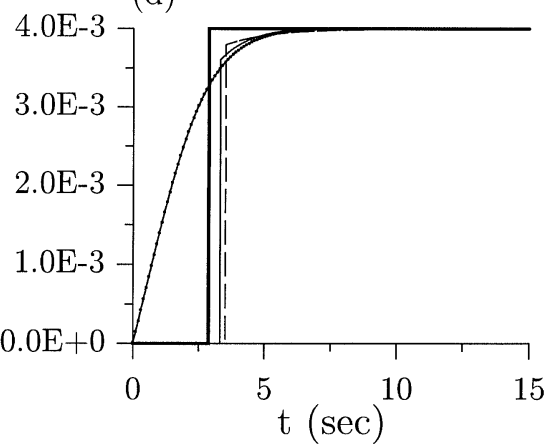

Figure 4. Comparison of the responses for four different initial stresses but with the same rectilinear strain path input: (a) time histories of the axial stress $\sigma_{11}$, (b) time histories of the shear stress $\sigma_{12}$, (c) paths of $\left(\sigma_{11}, \sigma_{12}\right)$, (d) time histories of $\dot{\lambda}$, (e) time histories of $\dot{\varepsilon}_{22}$. 


$$
\begin{aligned}
& \left(0, \frac{(2 v-1) \tau_{y} \dot{\varepsilon}_{12}}{(1+v) \sqrt{3 \dot{\varepsilon}_{11}^{2}+4 \dot{\varepsilon}_{12}^{2}}}\right),(0,300),(-300,0), \\
& \left(\frac{\sqrt{3} \tau_{y}}{\sqrt{(1+v)^{2} \dot{\varepsilon}_{11}^{2}+3 \dot{\varepsilon}_{12}^{2}}}, \frac{-(1+v) \tau_{y} \dot{\varepsilon}_{11}}{\sqrt{3} \dot{\varepsilon}_{12} \sqrt{(1+v)^{2} \dot{\varepsilon}_{11}^{2}+3 \dot{\varepsilon}_{12}^{2}}}\right),
\end{aligned}
$$

respectively. The first initial stress point was chosen such that the yield point was just the fixed point, whereas the last initial stress point was chosen to be located on the yield surface and to render $\dot{\lambda}\left(t_{i}\right)=0$. In the four examples the material constants were all taken to be $G=50000 \mathrm{MPa}, \tau_{y}=500 \mathrm{MPa}, v=0.3$. The resulting response paths are marked with path 1 , path 2, path 3, and path 4, respectively, in figure 4. It is interesting to observe that the four examples have the same limit strength points $\left(\bar{\sigma}_{11}, \bar{\sigma}_{12}\right)=\tau_{y}(3 / 2,-1 / 2)$. Figure $4 c$ shows that the limit strength point is asymptotically stable and figure $4 d$ shows that the corresponding $\dot{\lambda}$ tends to a fixed value. For the first example, path 1, the heavy lines in figures $4 a, 4 b$ and $4 c$ show that, once switched on, the stress components $\sigma_{11}$ and $\sigma_{12}$ do not vary again; that is, the point $\left(\bar{\sigma}_{11}, \bar{\sigma}_{12}\right)=\tau_{y}(3 / 2,-1 / 2)$ is a fixed point. However, for the other three examples, the stress paths tend to the fixed point very fast and get closer and closer to it as time elapses, but in fact it will take an infinite time to arrive at that point, so that in this sense the limit strength point is a limit point.

\subsection{Discontinuity of rate form contraction ratio}

In the limiting state $\dot{x}=0$, it follows from equations (3) $)_{1}$ and (50) that $\operatorname{tr} \dot{\varepsilon}=\left(1-2 v_{r}\right) \dot{\varepsilon}_{11}=0$; that is, the asymptotic value of the rate form contraction ratio is

$$
\bar{v}_{r}=\frac{1}{2} .
$$

However, $v_{r}$ is not always equal to $1 / 2$ in the plastic phase. This phenomenon is also reflected in the time histories of the hoop strain rates as shown in figure $4 e$ for the above four examples. The value $v_{r}$ may experience a jump from the elastic phase to the plastic phase as shown in figure $5 a$.

Substituting equation (5) into equation (17) we obtain

$$
3 K \operatorname{tr} \dot{\varepsilon}+\frac{E \dot{\lambda}}{3 \tau_{y}} \sigma_{11}=E \dot{\varepsilon}_{11}
$$

With the aid of this equation and definition $(2)_{1}$ the rate form contraction ratio is given by

$$
v_{r}=\frac{1}{6 K \dot{\varepsilon}_{11}}\left[(3 K-E) \dot{\varepsilon}_{11}+\frac{E}{3 \tau_{y}} \sigma_{11}(t) \dot{\lambda}(t)\right]=v+\frac{(1-2 v) \sigma_{11}(t) \dot{\lambda}(t)}{6 \tau_{y} \dot{\varepsilon}_{11}} .
$$

Due to the jump from $\dot{\lambda}=0$ in the elastic phase to the value $\dot{\lambda}>0$ in the plastic phase given by equation (23) ${ }_{1}$, $\operatorname{tr} \dot{\boldsymbol{\varepsilon}}$ may undergo a jump via equation (70), which thus leads to the jump of the rate form contraction ratio $v_{r}$,

$$
\left[v_{r}\right]:=v_{r}^{+}-v_{r}^{-}=v_{r}^{+}-v,
$$

where $v_{r}^{+}$and $v_{r}^{-}=v$ denote the rate form contraction ratios just after and before, respectively, the transition point from elasticity to plasticity. Using equations (71) and (23) we obtain 

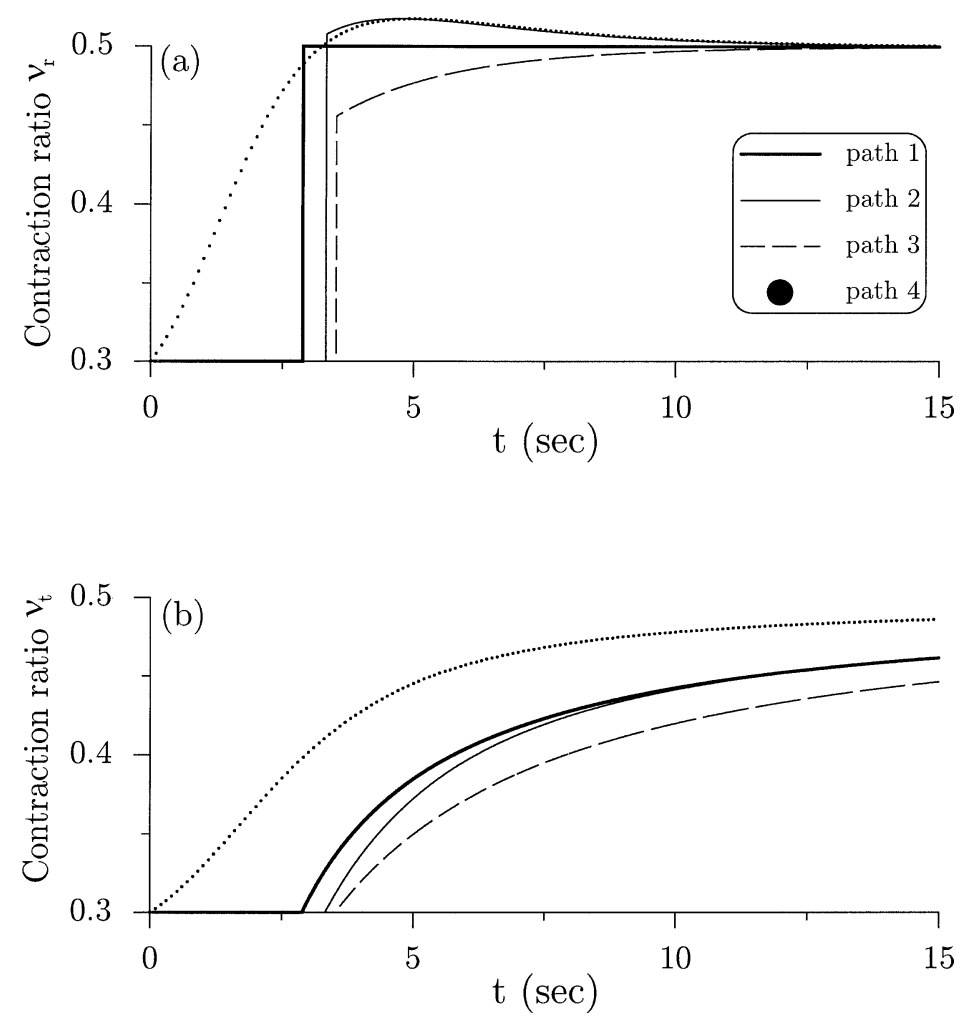

Figure 5. Comparison of the contraction ratios for four different initial stresses but with the same rectilinear strain path input: (a) time histories of the rate form contraction ratio $v_{r}$, (b) time histories of the total form contraction ratio $v_{t}$.

$$
\begin{aligned}
v_{r}^{+} & =\frac{1}{6 K \dot{\varepsilon}_{11}}\left[(3 K-E) \dot{\varepsilon}_{11}+\frac{E}{3 \tau_{y}} \sigma_{11}\left(t_{i}\right) \dot{\lambda}\left(t_{i}\right)\right] \\
& =\frac{1}{6 K \dot{\varepsilon}_{11}}\left[(3 K-E) \dot{\varepsilon}_{11}+\frac{E \sigma_{11}\left(t_{i}\right)\left(E \sigma_{11}\left(t_{i}\right) \dot{\varepsilon}_{11}+6 G \sigma_{12}\left(t_{i}\right) \dot{\varepsilon}_{12}\right)}{E \sigma_{11}^{2}\left(t_{i}\right)+9 G \sigma_{12}^{2}\left(t_{i}\right)}\right],
\end{aligned}
$$

where $t_{i}=t_{\text {on }}$ for the initiation of the plastic deformation. Obviously, when $\dot{\lambda}=0$ (the elastic phase) it leads to $v_{r}^{+}=v$. The value $\left[v_{r}\right]$ may be positive, zero, or negative as shown in figure $5 a$. For most cases it is positive or negative, and the rate form contraction ratio undergoes a discontinuity jump from $v$ to $v_{r}^{+}$. It needs to be pointed out that the value of $v_{r}$ may be greater than $1 / 2$ as shown by paths 2 and 4 in figure 4 . For these cases we have $\dot{\sigma}_{11}<0$ in some time intervals even when $\dot{\varepsilon}_{11}>0$. Wu (1996) recently studied torsional tests on cast and extruded aluminum, which results supported the current investigation that $v_{r}$ may be greater than $1 / 2$.

\subsection{Continuity of total form contraction ratio}

Integrating equation (5) from $t_{i}$ to $t$ and substituting equation $(3)_{2}$ for $\operatorname{tr} \boldsymbol{\varepsilon}(t)$, we obtain

$$
v_{t}=\frac{1}{2}-\frac{1}{2 \varepsilon_{11}(t)}\left[\operatorname{tr} \boldsymbol{\varepsilon}\left(t_{i}\right)+\varepsilon_{22}\left(t_{i}\right)-\varepsilon_{33}\left(t_{i}\right)+\frac{1}{3 K}\left(\sigma_{11}(t)-\sigma_{11}\left(t_{i}\right)\right)\right] .
$$

Obviously, it leads to $v_{t}^{+}=v$. Thus, $\left[v_{t}\right]=: v_{t}^{+}-v_{t}^{-}=v_{t}^{+}-v=0$, and so the total form contraction ratio experiences no discontinuity from the elastic phase to the plastic phase. The above equation indicates that $v_{t}$ 


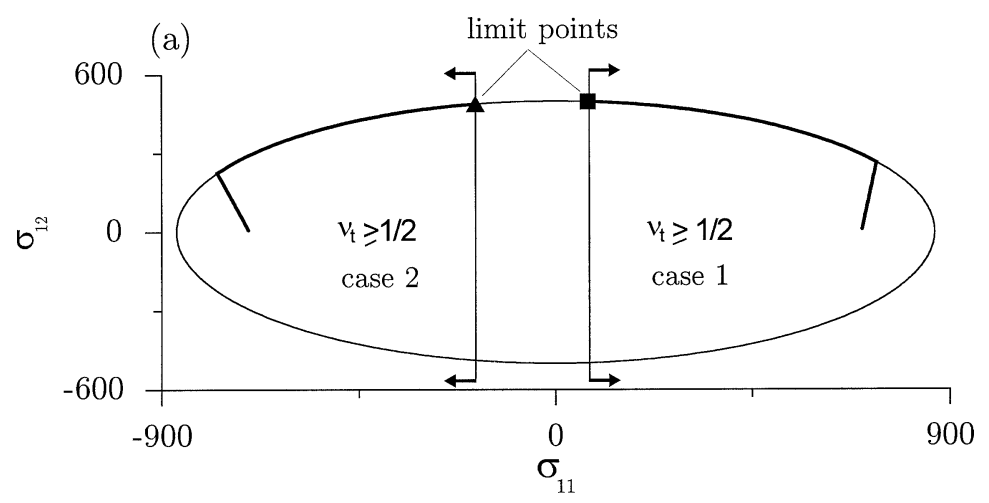

(a)

(b)

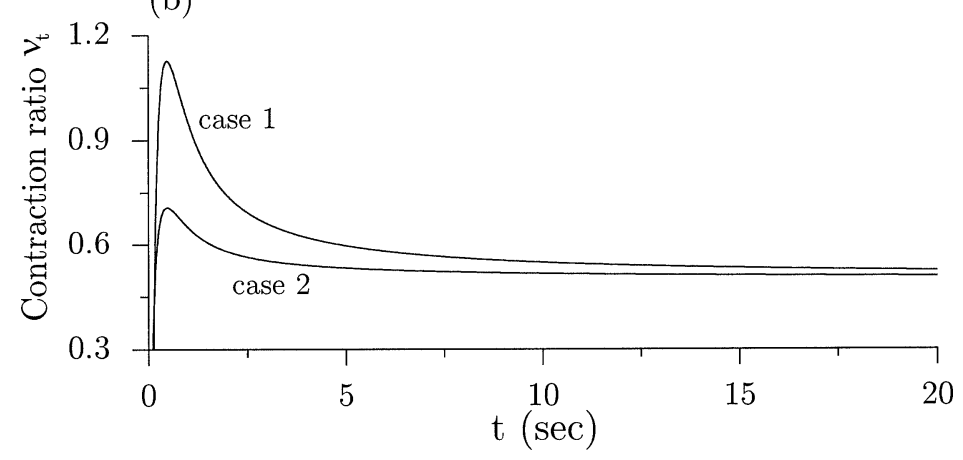

(b)

Figure 6. The total form contraction ratio $v_{t}$ may be greater than $1 / 2$. (a) The initial stresses which render $v_{t} \geqslant 1 / 2$ are marked, (b) time histories of $v_{t}$.

approaches $1 / 2$ asymptotically as $\varepsilon_{11}$ increases, as shown in figure $5 b$. Recall that the above is for the axialtorsional strain controls with rectilinear strain paths.

For simple tension or compression it is known that

$$
v_{t}=\frac{1}{2}-\left(\frac{1}{2}-v\right) \frac{\sigma_{11}}{E \varepsilon_{11}} .
$$

See, for example, Lemaitre and Chaboche (1985) and Khan and Huang (1995). The axial stress $\sigma_{11} \leqslant \sqrt{3} \tau_{y}$; thus, the total form contraction ratio $v_{t}$ approaches $1 / 2$ asymptotically as in the axial-torsional case. However, for the rate form contraction ratio, substituting $\sigma_{12}=0$ and $\dot{\varepsilon}_{12}=0$ into equation (71) and noting $\dot{\lambda}=$ $3 \tau_{y} \dot{\varepsilon}_{11} / \sigma_{11}$, we obtain $v_{r}=1 / 2$ for the simple tension or compression, which is different from the axialtorsional case, for which $v_{r}=1 / 2$ holds only in the limiting state.

In view of equation (75) it should be $v_{t}<1 / 2$ for simple tension or compression because $v<1 / 2$. But, for the axial-torsional case, some pre-stress conditions may render $v_{t} \geqslant 1 / 2$. For demonstration, let us consider the initial stress $\left(\sigma_{11}\left(t_{i}\right), \sigma_{12}\left(t_{i}\right)\right)$ which is confined by the inequality $\sigma_{11}^{2}\left(t_{i}\right)+3 \sigma_{12}^{2}\left(t_{i}\right) \leqslant 3 \tau_{y}^{2}$. Upon straining along the path (59) with $\varepsilon_{11}\left(t_{i}\right)=\varepsilon_{12}\left(t_{i}\right)=0$ and $\varepsilon_{22}\left(t_{i}\right)=\varepsilon_{33}\left(t_{i}\right)$, we have

$$
\sigma_{11}\left(t_{\mathrm{on}}\right)=\sigma_{11}\left(t_{i}\right)+3 K(1-2 v) \varepsilon_{11}\left(t_{\mathrm{on}}\right)
$$



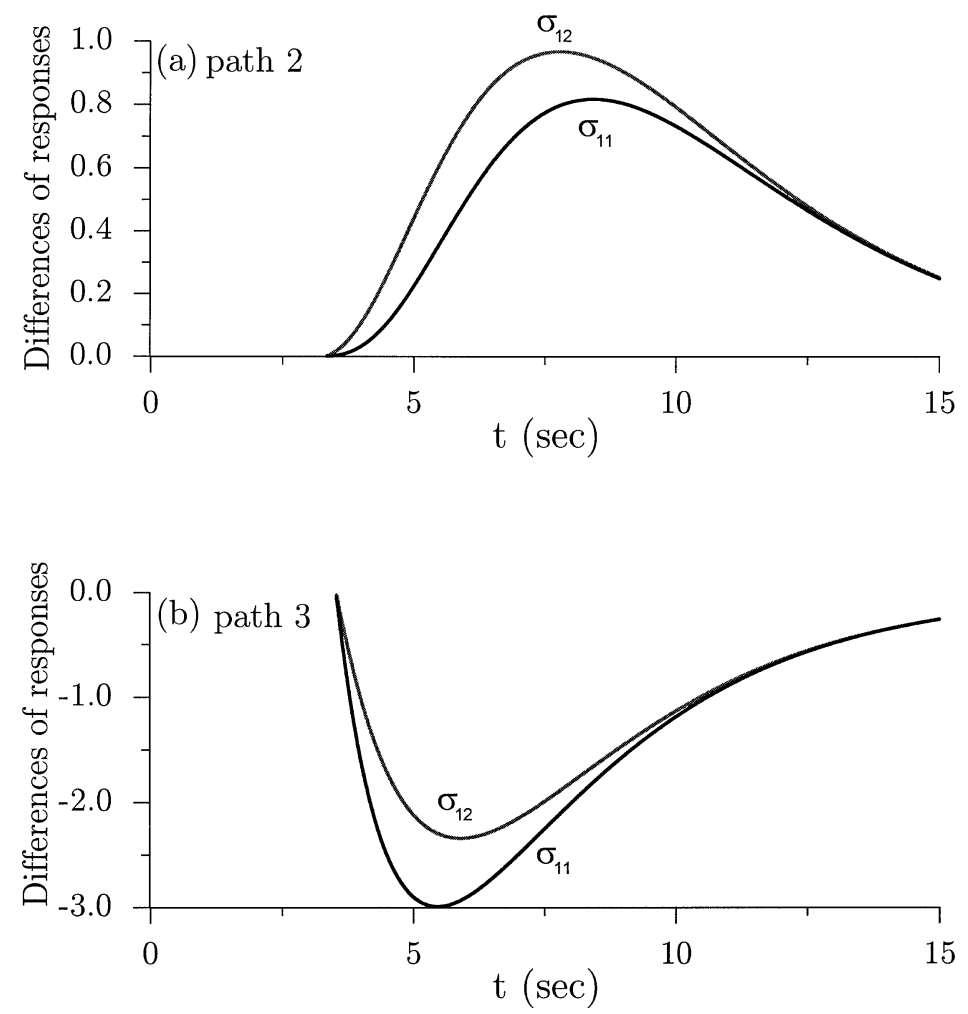

Figure 7. The differences of the responses $\sigma_{11}$ and $\sigma_{12}$ between the exact one and the one with the assumption $v_{r}=1 / 2$ : (a) path 2, (b) path 3 .

Setting $t_{i}=t_{\mathrm{on}}$ in equation (74), substituting the above equation for $\sigma_{11}\left(t_{\mathrm{on}}\right)$, and noting $\operatorname{tr} \boldsymbol{\varepsilon}\left(t_{\mathrm{on}}\right)=(1-$ $2 v) \varepsilon_{11}\left(t_{\mathrm{on}}\right)$, we obtain

$$
v_{t}=\frac{1}{2}-\frac{1}{6 K \varepsilon_{11}(t)}\left[\sigma_{11}(t)-\sigma_{11}\left(t_{i}\right)\right] \quad \forall t \geqslant t_{\mathrm{on}} .
$$

Depending on $\left[\sigma_{11}(t)-\sigma_{11}\left(t_{i}\right)\right] / \varepsilon_{11}(t)$, the value $v_{t}$ may be greater than $1 / 2$. Recall that the limit strength as given in equation (66) is the limit value of $\sigma_{11}$ and $\sigma_{12}$ when $t$ approaches infinity. Thus, when

$$
\sigma_{11}\left(t_{i}\right) \geqslant \frac{3 \tau_{y} \dot{\varepsilon}_{11}}{\sqrt{3 \dot{\varepsilon}_{11}^{2}+4 \dot{\varepsilon}_{12}^{2}}}, \quad \dot{\varepsilon}_{11}>0
$$

or when

$$
\sigma_{11}\left(t_{i}\right) \leqslant \frac{3 \tau_{y} \dot{\varepsilon}_{11}}{\sqrt{3 \dot{\varepsilon}_{11}^{2}+4 \dot{\varepsilon}_{12}^{2}}}, \quad \dot{\varepsilon}_{11}<0,
$$

it should be $v_{t} \geqslant 1 / 2$; see figure $6 a$, where the region with $v_{t} \geqslant 1 / 2$ is marked. Two cases are plotted in figure $6 b$ with initial stresses $\sigma_{11}\left(t_{i}\right)=700 \mathrm{MPa}$ and $\sigma_{12}\left(t_{i}\right)=0 \mathrm{MPa}$, and strain rates $\dot{\varepsilon}_{11}=0.002 \mathrm{~s}^{-1}$ and $\dot{\varepsilon}_{12}=0.02 \mathrm{~s}^{-1}$ for case 1 , and $\sigma_{11}\left(t_{i}\right)=-700 \mathrm{MPa}$ and $\sigma_{12}\left(t_{i}\right)=0 \mathrm{MPa}$, and $\dot{\varepsilon}_{11}=-0.005 \mathrm{~s}^{-1}$ and $\dot{\varepsilon}_{12}=0.02 \mathrm{~s}^{-1}$ for case 2 . 
(a)
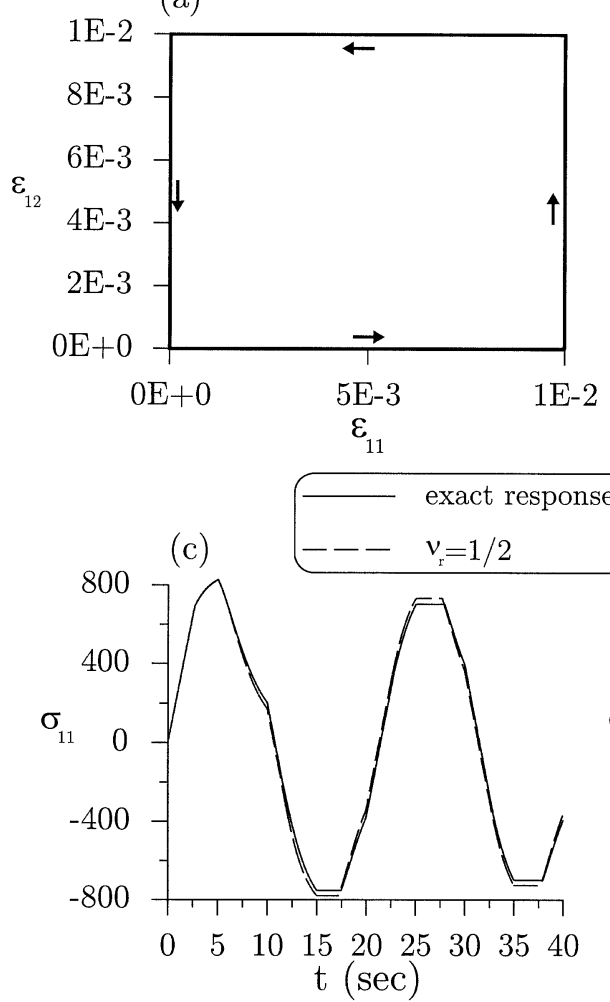

(b)

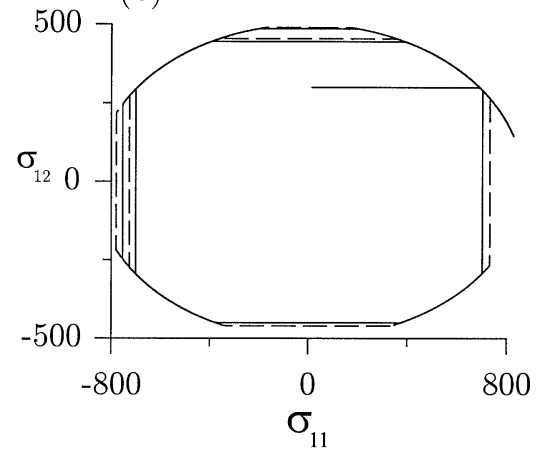

(d)

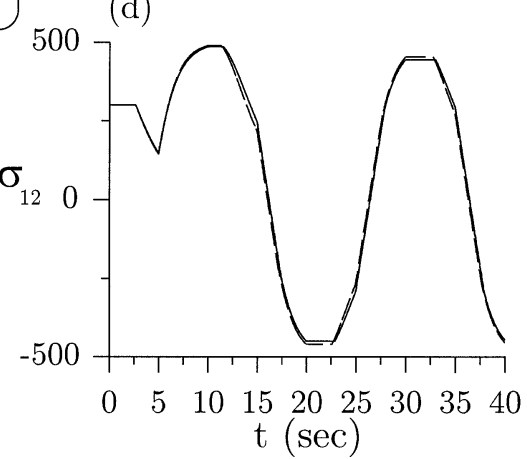

Figure 8. Comparison of the responses $\sigma_{11}$ and $\sigma_{12}$ between the exact one and the one with the assumption $v_{r}=1 / 2$ for a rectangular strain path input: (a) input strain path, (b) output stress paths, (c) time histories of $\sigma_{11}$, (d) time histories of $\sigma_{12}$.

5.6. Validity of the assumption $v_{r}=1 / 2$

Under the assumption $v_{r}=1 / 2, \dot{\mathbf{q}}$ in equation (32) reduces to

$$
\dot{\mathbf{q}}=\left[\begin{array}{c}
\sqrt{3} \dot{\varepsilon}_{11} \\
2 \dot{\varepsilon}_{12}
\end{array}\right]
$$

and equation (48) changes to

$$
\dot{x}=a+b \sinh x,
$$

where

$$
a:=\frac{G}{\sqrt{3} \tau_{y}^{2}}\left(2 \sigma_{11}\left(t_{i}\right) \dot{\varepsilon}_{12}-3 \sigma_{12}\left(t_{i}\right) \dot{\varepsilon}_{11}\right), \quad b:=-\frac{G}{\tau_{y}^{2}}\left(\sigma_{11}\left(t_{i}\right) \dot{\varepsilon}_{11}+2 \sigma_{12}\left(t_{i}\right) \dot{\varepsilon}_{12}\right) .
$$

The solution of equation (79) is

$$
a \tanh \frac{x}{2}=b+\sqrt{a^{2}+b^{2}} \tanh \left[\operatorname{arth}\left(\frac{-b}{\sqrt{a^{2}+b^{2}}}\right)+\frac{\sqrt{a^{2}+b^{2}}}{2}\left(t-t_{i}\right)\right] .
$$

According to this formula the responses can be calculated and compared with the results calculated from equation (52); figure 7 shows that the differences of the responses are appreciable in the initial stage of the 
plastic deformation; however, for large deformation the differences gradually diminish to zero. Figure 8 also shows the comparison between the exact one and the one with the above assumption $v_{r}=1 / 2$, but the input strain control is a rectangular path.

\section{Concluding remarks}

To analyse thin-walled tubes under combined axial and torsional controls, which lead to highly nonlinear differential equations, we proposed a new formulation in which the stresses were parametrized and the consistency condition and initial condition were fulfilled automatically. Upon solving the first order differential equation for the parameter $x$, we can readily calculate the stresses, and also the hoop and radial strains. For mixed controls, we can directly obtain the responses without solving the first-order differential equation.

With the new formulation we investigated the rate form and total form contraction ratios in the thin-walled tube, showing that the rate form contraction ratio may undergo a discontinuity jump in the transition point of elasticity-plasticity and that, depending on the control paths and on the initial stresses, the jump quantity may be positive, zero, or negative. The value of the rate form contraction ratio may be greater than $1 / 2$, but it eventually tends to $1 / 2$ in the limiting state. For the total form contraction ratio, there exists no similar jump in the elasticity-plasticity transition point; however, its value may still be greater than $1 / 2$ and then approach $1 / 2$ in the limiting state. The conventional simplified assumption of $v_{r}=1 / 2$ may lead to appreciable errors in the initial stage of plastic deformation; it is acceptable only in the limiting state.

\section{References}

Hill R., The Mathematical Theory of Plasticity, Oxford University Press, New York, 1950.

Hong H.-K., Liu C.-S., Prandtl-Reuss elastoplasticity: On-off switch and superposition formulae, Int. J. Solids Struct. 34 (1997) $4281-4304$.

Hong H.-K., Liu C.-S., On behavior of perfect elastoplasticity under rectilinear paths, Int. J. Solids Struct. 35 (1998) 3539-3571.

Hong H.-K., Liu C.-S., Internal symmetry in the constitutive model of perfect elastoplasticity, Int. J. Non-Linear Mechs. 35 (2000) $447-466$.

Khan A.S., Huang S., Continuum Theory of Plasticity, Wiley, New York, 1995.

Klisinski M., Mroz Z., Runesson K., Structure of constitutive equations in plasticity for different choices of state and control variables, Int. J. Plasticity 8 (1992) 221-243.

Lemaitre J., Chaboche J.L., Mechanics of Solids Materials, Cambridge University Press, Cambridge, 1985.

Nadai A., Theory of Flow and Fracture of Solids, McGraw-Hill, New York, 1950.

Prandtl L., Spannungsverteilung in plastischen kœrpern, in: Proceedings of the 1st International Congress on Applied Mechanics, Delft, 1924, pp. 43-54.

Reuss E., Beruecksichtigung der elastischen formaenderungen in der plastizitaetstheorie, Z. Angew. Math. Mech. (ZAMM) 10 (1930) $266-274$.

Wu H.C., The torsion test and its role in constitutive equations for metals, Chinese J. Mech. 12 (1996) 121-130. 